\title{
抗衡离子对硫酸盐表面活性剂气/液界面性质影响的分子动力学模拟
}

\author{
胡松青 ${ }^{1, *} \quad$ 纪贤晶 ${ }^{1} \quad$ 范忠钰 $^{2}$ 张田田 ${ }^{1} \quad$ 孙霜青 ${ }^{1}$ \\ ('中国石油大学(华东)理学院, 山东青岛 266580；2华北油田公司勘探开发研究院,河北任丘 062552)
}

\begin{abstract}
摘要: 采用全原子分子动力学方法研究了抗衡离子为第一主族离子 $\left(\mathrm{Li}^{+} 、 \mathrm{Na}^{+} 、 \mathrm{~K}^{+} 、 \mathrm{Rb}^{+}\right.$和 $\left.\mathrm{Cs}^{+}\right)$的十二烷基硫酸 盐表面活性剂的气/液界面性质. 通过分析体系中各组分的密度分布曲线, 考察表面活性剂单分子层在界面的 聚集形态, 并利用径向分布函数分析了表面活性剂极性头基与抗衡离子间的相互作用. 研究结果表明: 随着抗 衡离子半径的增大, 不同体系的界面水层厚度依次增加, 表面活性剂极性头基与抗衡离子形成的 Stern 和扩散 层厚度也相应增加. 但表面活性剂吸附层的抗衡离子缔合度以及体系表面张力却随抗衡离子半径的增大而减 小. 研究表明抗衡离子的差异对十二烷基硫酸盐表面活性剂气/液界面性质有很大影响.
\end{abstract}

关键词: 十二烷基硫酸盐; 抗衡离子; 气/液界面; 双电层; 分子动力学 中图分类号: 0641; 0647

\section{Counterion Effects on the Properties of Sulfate Surfactants at the Air/Liquid Interface by Molecular Dynamics Simulation}

\author{
HU Song-Qing, $\quad$ JI Xian-Jing1 $\quad$ FAN Zhong-Yu ${ }^{2} \quad$ ZHANG Tian-Tian ${ }^{1} \quad$ SUN Shuang-Qing ${ }^{1}$ \\ ('College of Science, China University of Petroleum (East China), Qingdao 266580, Shandong Province, P. R. China; \\ ${ }^{2}$ Exploration and Development Research Institute, Huabei Oilfield Company, Renqiu 062552, Hebei Province, P. R. China)
}

\begin{abstract}
Molecular dynamics (MD) simulations have been carried out to study the micro-interfaces between counterions $\left(\mathrm{Li}^{+}, \mathrm{Na}^{+}, \mathrm{K}^{+}, \mathrm{Rb}^{+}, \mathrm{Cs}^{+}\right)$and dodecyl sulfate surfactant at the air/liquid interface. The morphology of the monolayer was analyzed by the density profile of the surfactant components, and the interactions between the polar head groups of the surfactants and the counterions were analyzed using the radial distribution function (RDF). The results showed that the interfacial thickness and thicknesses of the Stern and diffusion layers increased as the radius of counterion increased, whereas the surface tension and the degree of counterion association at the adsorbed layer decreased as the radius increased. These results indicated that the different counterions had a significant effect on the adsorption behavior of the dodecyl sulfate surfactants at the interface.
\end{abstract}

Key Words: Dodecyl sulfate; Counterion; Air/liquid interface; Electrical double layer;

Molecular dynamics

\section{1 引言}

阴离子表面活性剂以其优异的性能被广泛应 用于日常生活和工业生产中, 如洗涤剂、食品添加 剂、发泡剂、杀菌剂等. ${ }^{1-4}$ 阴离子型表面活性剂在界 面的吸附是其发挥效用的关键, 且与分子结构紧密
相关. 除亲水基和疏水基外, 抗衡离子对阴离子表 面活性剂在界面的吸附行为也有较大影响..$^{5-11}$ 现有 的实验手段如小角度中子散射、拉曼光谱、X射线衍 射等被广泛应用于抗衡离子对表面活性剂气/液界 面性质影响的研究中. Wang 和 Morgner ${ }^{12}$ 利用中性

Received: October 14, 2014; Revised: November 19, 2014; Published on Web: November 19, 2014.

"Corresponding author. Email: ccupc@163.com; Tel: +86-532-86983170.

The project was supported by the National Natural Science Foundation of China (51201183), Promotive Research Fund for Excellent Young and Middle-Aged Scientists of Shandong Province, China (BS2013CL033), and CNPC Innovation Foundation, China (2011D-5006-0202).

国家自然科学基金(51201183)，山东省优秀中青年科学家基金(BS2013CL033)和中国石油科技创新基金(2011D-5006-0202)资助项目

(c) Editorial office of Acta Physico-Chimica Sinica 
离子碰撞散射谱研究了抗衡离子 $\mathrm{Na}^{+} 、 \mathrm{Cs}^{+}$对十二烷 基硫酸盐表面活性剂的表面张力和界面双电层结 构的影响, 发现抗衡离子大小和溶解度对其在气/液 界面的吸附有较大影响. Bordes 等 ${ }^{13}$ 通过电导率法 考察了抗衡离子 $\mathrm{Li}^{+} 、 \mathrm{Na}^{+} 、 \mathrm{~K}^{+}$对氨基酸类表面活性剂 溶液导电性能的影响, 结果表明 $\mathrm{Li}^{+}$与氨基酸类表面 活性剂胶束结合最为稳定. 2004 年, Gilányi 等 ${ }^{14}$ 通过 测量吸附等温线考察了十二烷基硫酸碱盐 $\mathrm{Li}^{+} 、 \mathrm{Na}^{+}$、 $\mathrm{K}^{+} 、 \mathrm{Rb}^{+} 、 \mathrm{Cs}^{+}$的气/液界面性质, 根据古埃-查普曼-斯 特恩理论提出了新的模型用于解释阴离子表面活 性剂在界面的吸附行为.

近年来, 采用计算机分子模拟技术在分子原子 水平上研究表面活性剂溶液性质的报道越来越多. Rakitin 等 ${ }^{15}$ 利用分子动力学模拟研究了抗衡离子 $\mathrm{Li}^{+} 、 \mathrm{Na}^{+} 、 \mathrm{NH}_{4}^{+}$对十二烷基硫酸盐表面活性剂溶液中 胶束结构的影响, 结果发现, 随着离子半径的增加, 抗衡离子进入胶束结构的能力减弱. Hantal 等 ${ }^{16}$ 采用 分子动力学方法研究抗衡离子 $\left(\mathrm{Li}^{+} 、 \mathrm{Na}^{+} 、 \mathrm{~K}^{+} 、 \mathrm{Rb}^{+} 、\right.$ $\mathrm{Cs}^{+}$)在两种表面浓度下对癸烷基硫酸盐表面活性剂 吸附层的影响, 并验证了所使用的吸附理论的正确 性. 十二烷基硫酸盐是最常见的表面活性剂, 但抗 衡离子影响其气/液界面性质的模拟研究鲜有报道. 因此, 本文以十二烷基硫酸盐为研究对象, 采用全 原子分子动力学方法, 考察第一主族碱金属离子 $\left(\mathrm{Li}^{+} 、 \mathrm{Na}^{+} 、 \mathrm{~K}^{+} 、 \mathrm{Rb}^{+} 、 \mathrm{Cs}^{+}\right)$对其气/液界面性质的影响规 律, 获得抗衡离子与表面活性剂微观作用机理.

\section{2 计算方法和模型}

首先利用 Visualizer 模块构建水分子和十二烷 基硫酸盐表面活性剂分子, 选择 COMPASS 力场 ${ }^{17}$ 进行计算, 并采用 Smart Minimizer 对分子进行初步 优化, 以消除搭建构型过程中可能出现的分子重 叠. 其次, 构建尺寸为 $4 \times 4$ 的包含 16 个 $^{18,19}$ 十二烷基 硫酸盐单分子层, 并使表面活性剂单分子垂直于 $x$ 、 $y$ 轴组成的平面. 其中, 每个表面活性剂分子的占有 面积为 $0.55 \mathrm{~nm}^{2}$, 与 Tajima 等 ${ }^{20}$ 实验测得的数据相 近. 构建完单层后, 把表面活性剂极性头插入含 900 个水分子的厚度为 $3 \mathrm{~nm}$ 的水层表面, 水分子采用 SPC 势能模型. 有文献 ${ }^{21}$ 表明 $2 \mathrm{~nm}$ 厚度的水层就足 以保证水层中的水分子性质接近本体溶液, 同时也 足以保证两个表面活性剂单层相互独立. 紧接着随 机向水盒子中加入与表面活性剂同等数量的抗衡 离子 $\left(\mathrm{Li}^{+} 、 \mathrm{Na}^{+} 、 \mathrm{~K}^{+} 、 \mathrm{Rb}^{+} 、 \mathrm{Cs}^{+}\right)$, 以保证体系的电中性.
所有模型均采用三维周期性结构, 以消除边界效应 的影响. 为减小 $z$ 方向上的周期性重复对体系的影 响, 在体系两侧分别加厚度为 $3 \mathrm{~nm}$ 的真空层, 详细 的模型信息见表 1 .

全部模拟计算采用 Accerlrys 公司的 Materials Studio 软件完成, 并选择 NVT 系综对分子动力学模 拟计算进行限制. 采用Andersen 控温方法 22 将温度 控制在 298 K. 各分子的起始速度由 MaxwellBoltzmann 分布随机产生, 运用 velocity Verlet算法 ${ }^{23}$ 解牛顿运动方程. 长程静电相互作用选择 Ewald 方 法 ${ }^{24}$ 进行计算, 范德瓦尔兹相互作用选择 atom based 方法计算, 截断半径为 $0.95 \mathrm{~nm}$, 截断距离之外的分 子间相互作用能按平均密度近似方法进行校正. 模 拟中时间步长选择 $1 \mathrm{fs}$, 每 $1 \mathrm{ps}$ 记录一次轨迹信息, 模拟总耗时 $4 \mathrm{~ns}$, 最后 $1 \mathrm{~ns}$ 存储的轨迹文件用于后 续结构和动力学分析.

\section{3 结果和讨论}

\section{1 单分子层的结构性质}

以十二烷基硫酸铯(CDS)为例讨论表面活性剂 单分子层的结构性质. 图 1(a)和(b)分别为十二烷基 硫酸铯(CDS)体系的初始构型和平衡构型. 从图中可 以发现, 经过纳米级的模拟时间后, 表面活性剂分子 在气/液界面处发生聚集, 形成稳定单层膜结构. 其 中, 表面活性剂亲水头基伸向水相, 而疏水烷基链则 向气相延伸. 抗衡离子吸附在表面活性剂头基周围, 但也有少数抗衡离子游离于体相水中. 此外, 模拟中 还发现有极少量的水分子进入表面活性剂单分子 层. 这与实验得到的表面活性剂在气/液界面的分布 情况一致, 25,26 表明采用分子动力学模拟方法研究表 面活性剂在气/夜界面的吸附具有一定的可行性.

通过研究体系中各组分的密度分布函数可以获 得表面活性剂单分子层的详细结构特征. 图 2 为不

表 1 各体系的具体模型参数

Table 1 Detailed model parameters of different systems

\begin{tabular}{ccccc}
\hline System & Box dimension $/ \mathrm{nm}^{3}$ & Counterion & $\begin{array}{c}\text { Number of } \\
\text { counterions }\end{array}$ & $\begin{array}{c}\text { Number of } \\
\text { water molecules }\end{array}$ \\
\hline LDS & $2.91 \times 2.91 \times 13.84$ & $\mathrm{Li}^{+}$ & 32 & 900 \\
SDS & $2.91 \times 2.91 \times 13.85$ & $\mathrm{Na}^{+}$ & 32 & 900 \\
PDS & $2.91 \times 2.91 \times 13.89$ & $\mathrm{~K}^{+}$ & 32 & 900 \\
RDS & $2.91 \times 2.91 \times 13.87$ & $\mathrm{Rb}^{+}$ & 32 & 900 \\
CDS & $2.91 \times 2.91 \times 13.84$ & $\mathrm{Cs}^{+}$ & 32 & 900 \\
\hline
\end{tabular}

LDS: lithium dodecyl sulfate; SDS: sodium dodecyl sulfate; PDS: potassium dodecyl sulfate; RDS: rubidium dodecyl sulfate; CDS: cesium dodecyl sulfate 


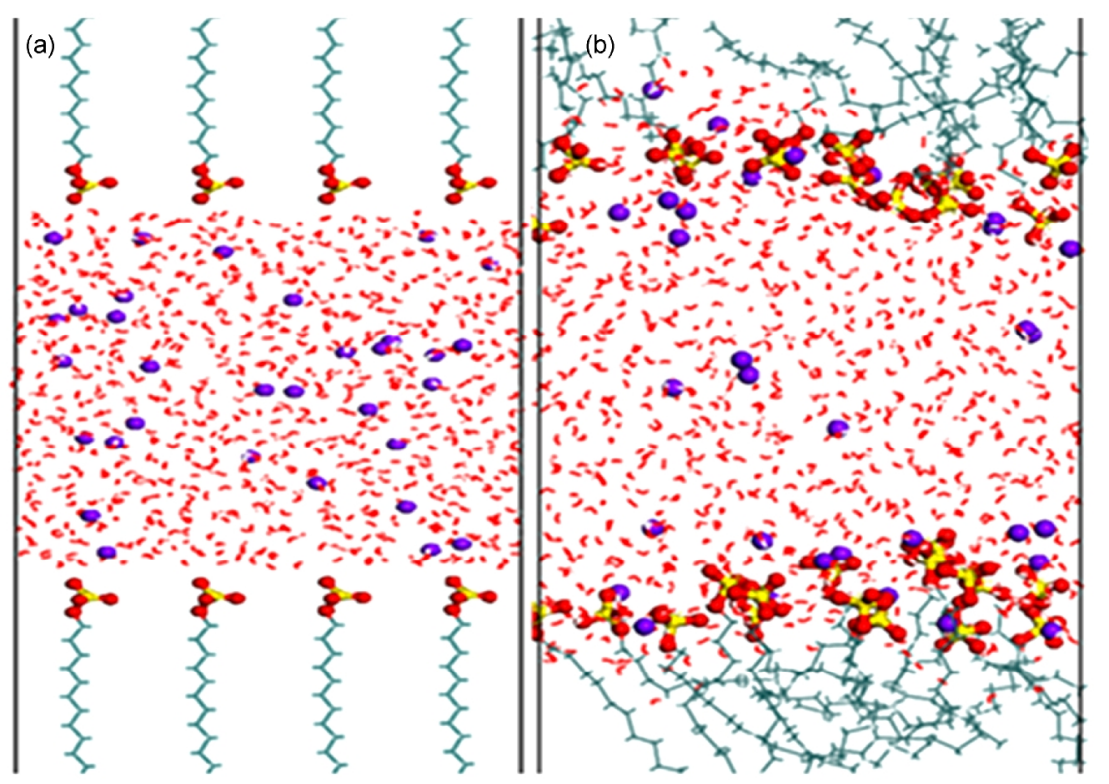

图 $1 \mathrm{CDS}$ 体系的初始(a)和平衡(b)构型

Fig.1 Initial (a) and balanced (b) configurations for CDS system

同十二烷基硫酸盐/抗衡离子体系中各组分(烷基链、 极性头基、水和抗衡离子)在 $z$ 方向上的密度分布曲 线(体系中心为横坐标起点). 从图中可以看出, 表面 活性剂头基(峰值范围 0.8-2.0 nm) 处于水相中(峰值 范围 0-2.3 nm), 这表明 $-\mathrm{SO}_{4}$ 头基进入到水层发生 水化作用. 在距离体系中心 $1.5-2.3 \mathrm{~nm}$ 的范围内, 水 相密度和头基密度均逐渐降低, 这表明沿 $z$ 轴方向表 面活性剂头基的水化作用逐渐降低. 此外, 带正电的
抗衡离子(峰值范围 0.8-1.5 nm) 分布在带负电的头 基周围(峰值范围 0.8-2.0 nm)内, 说明由于静电相互 作用, 抗衡离子与表面活性剂极性头基结合.

图 2 中另一个显著的特点是: 在距离体系中心 1.0-2.5 nm 的范围内, 存在一个明显的界面水分子 层. 本节采用 “ $10 \%-90 \%$ 厚度原则”, ${ }^{27}$ 即体相水密度 由 $10 \%$ 到 $90 \%$ 的变化区间, 计算 LDS、SDS、PDS、 $\mathrm{RDS}$ 和 CDS 体系的界面水层厚度分别为 0.808 、
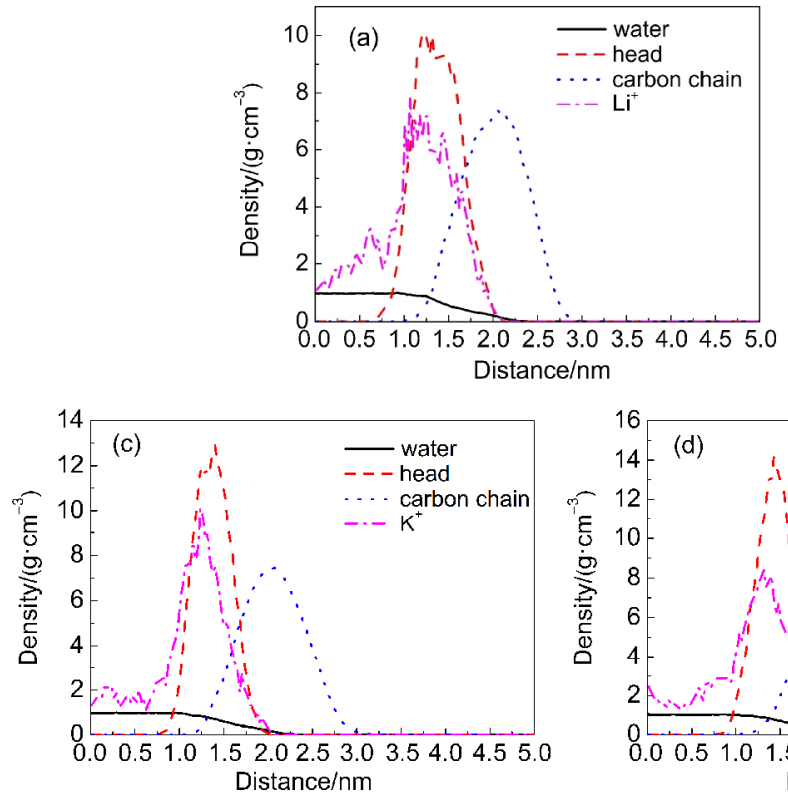

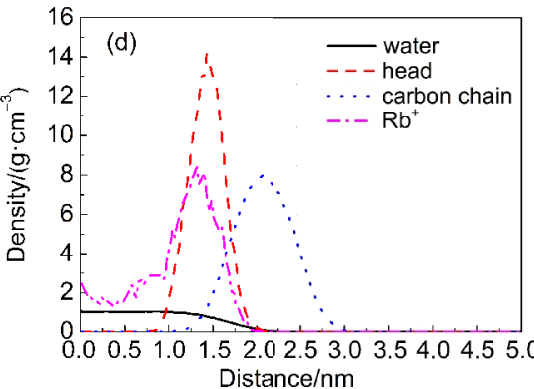

图 2 各组分密度分布曲线

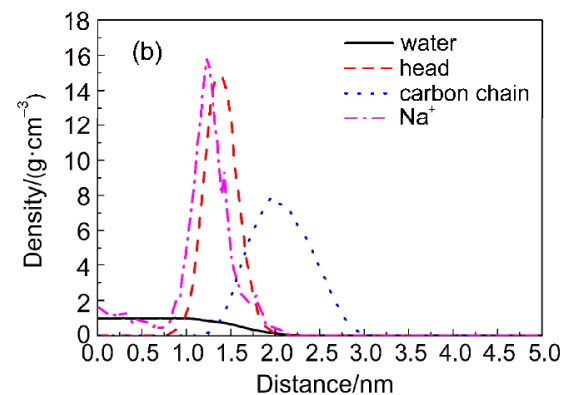

Fig.2 Density profiles of different components

(a) LDS; (b) SDS; (c) PDS; (d) RDS; (e) CDS

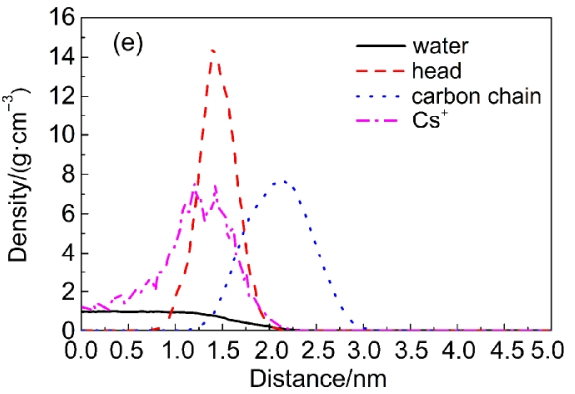

Distance/nm 
$0.815 、 0.903 、 0.928$ 和 $1.016 \mathrm{~nm}$. 即各体系的界面水 层厚度由小到大的顺序为 $\mathrm{LDS}<\mathrm{SDS}<\mathrm{PDS}<\mathrm{RDS}<$ CDS. 抗衡离子半径越大, 体系界面水层厚度越大. 这是因为抗衡离子的半径越大, 在溶液中的迁移能 力越弱, 对极性头基与水分子间的屏蔽作用越小, 从 而导致该体系中表面活性剂头基与水分子的相互作 用强于其它同主族抗衡离子, 界面水层厚度增大.

\section{2 抗衡离子与极性头基间的相互作用}

\subsection{1 双电层的结构性质}

阴离子表面活性剂在气/液界面吸附时, 溶解在 水中的抗衡离子与表面活性剂会形成界面双电层 结构, 且双电层的结构性质对研究表面活性剂极性 头基与抗衡离子之间的相互作用极其重要. 因此, 本节利用径向分布函数(RDF)考察表面活性剂头基 和抗衡离子形成的界面双电层结构. ${ }^{28}$ 图 3 所示为表 面活性剂头基中硫原子和抗衡离子间的径向分布 函数 $g_{\mathrm{S}-\mathrm{M}^{+}}(r)$.

在图 3 中, 以十二烷基硫酸铯(CDS)为例, 发现 头基和抗衡离子 $\mathrm{Cs}^{+}$通过静电相互作用形成了结构 良好的径向分布函数曲线. 在 $0.376 \mathrm{~nm}$ 位置出现第 一峰, 第一峰可代表 Stern 层, 且第一峰起始位置在 $0.326 \mathrm{~nm}$ 处, 终止位置在 $0.489 \mathrm{~nm}$ 处, 即 Stern 层厚 度为 $0.163 \mathrm{~nm}(0.489-0.326 \mathrm{~nm})$. 另外, 在 $0.598 \mathrm{~nm}$ 处出现第二个相对较弱的峰代表扩散层, 且扩散层 厚度为 $0.252 \mathrm{~nm}(0.741-0.489 \mathrm{~nm})$. 对比不同体系 头基和抗衡离子间的径向分布函数, 从 LDS 到 CDS 头基和抗衡离子聚集峰的强度逐渐减弱, 峰的位置 依次右移. 表 2 中列出不同十二烷基硫酸盐/抗衡离 子体系的 Stern层和扩散层厚度. 从表中可以看出, 不同体系的 Stern 层和扩散层厚度满足顺序: LDS $<$

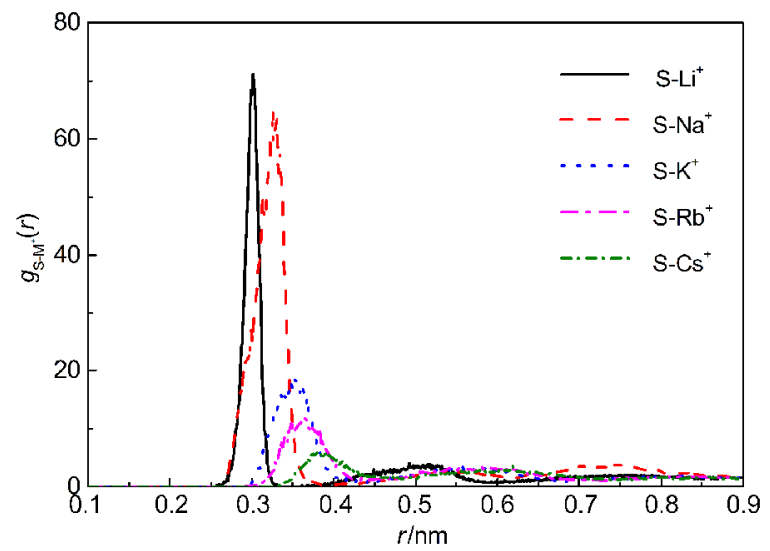

图3 表面活性剂头基和抗衡离子的径向分布函数

Fig.3 Radial distribution function (RDF) between the head group of surfactant and counterions
$\mathrm{SDS}<\mathrm{PDS}<\mathrm{RDS}<\mathrm{CDS}$, 即随抗衡离子半径的增加, 体系的 Stern 层和扩散层厚度相应增加. 这主要是因 为随着抗衡离子半径增加, 抗衡离子进入界面层的 能力减弱, 抗衡离子与表面活性剂头基距离增加, 且抗衡离子和头基之间的静电吸引作用减小, 头基 对抗衡离子的束缚作用减弱, 从而使 Stern 层和扩散 层厚度增加.

\subsection{2 吸附层的抗衡离子缔合度}

离子型表面活性剂在界面处发生吸附时, 由于 静电相互作用, 部分抗衡离子会与带相反电荷的表 面活性剂极性头基结合形成缔合结构. 图 4 所示为 不同体系中表面活性剂吸附层的抗衡离子缔合结 构. 平均每个表面活性剂离子所缔合的抗衡离子数 目即为表面活性剂吸附层的抗衡离子缔合度, ${ }^{29,30}$ 用 $N_{0}$ 表示:

$$
N_{0}=\frac{N_{\mathrm{f}}}{K_{0}}
$$

式中, $N_{\mathrm{f}}$ 表示与表面活性剂头基缔合的抗衡离子数 目, $K_{0}$ 代表体系中表面活性剂数目. 据模拟结果获 得的不同体系吸附层的抗衡离子缔合度 $N_{0}$ 列于表 3. 从表中可以看出, 不同体系吸附层的抗衡离子缔 合度 $N_{0}$ 变化规律为: $\mathrm{LDS}>\mathrm{SDS}>\mathrm{PDS}>\mathrm{RDS}>\mathrm{CDS}$, 即 吸附层的抗衡离子缔合度随抗衡离子半径的增加 而降低. 这主要是由于抗衡离子半径增加, 抗衡离 子与表面活性剂头基距离增加, 静电相互作用减

表 2 不同体系Stern层和扩散层厚度

Table 2 Thicknesses of stern layer and diffusion layer in different systems

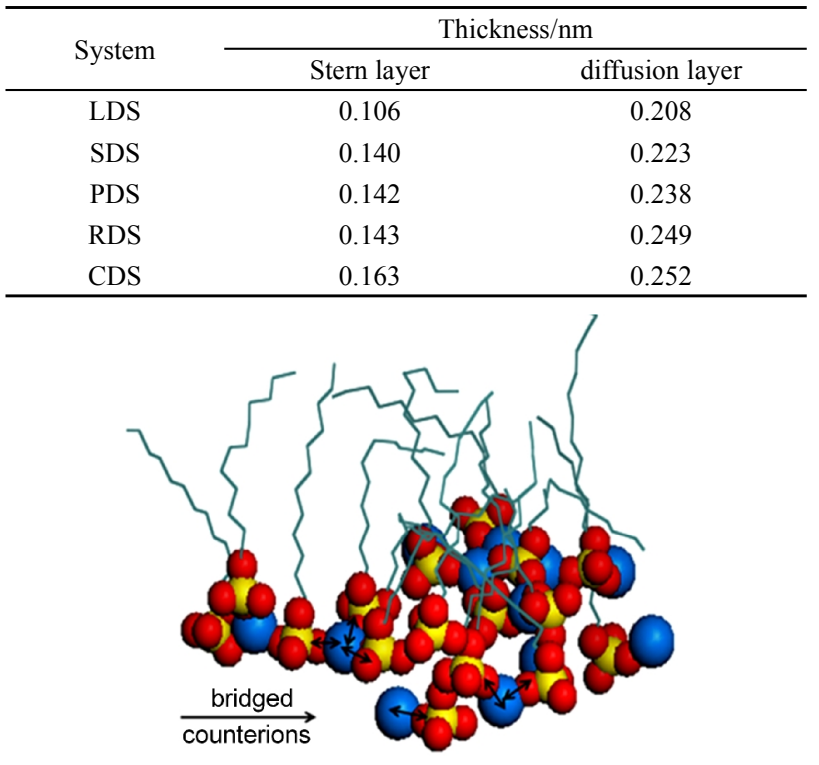

图 4 吸附层的抗衡离子缔合结构

Fig.4 Structure of bridged counterions in adsorbed layer 
小, 溶液中的抗衡离子向界面处迁移的能力减弱, 因此吸附层的抗衡离子缔合度降低.

\section{3 极性头基和水分子的相互作用}

表面活性剂极性头基与水分子间的相互作用 对表面活性剂的吸附行为具有重要的影响. 为深入 研究抗衡离子对阴离子表面活性剂气/液界面吸附 行为的影响, 本节计算了不同十二烷基硫酸盐/抗衡 离子体系的极性头基中氧原子 $\left(\mathrm{O}_{\mathrm{h}}\right)$ 与水中氧原子 $\left(\mathrm{O}_{\mathrm{w}}\right)$ 之间的径向分布函数 $g_{\mathrm{O}_{\mathrm{n}} \mathrm{O}_{\mathrm{w}}}(r)$, 如图 5 所示. 从图 中可看出, 在 5 种体系中, 水中氧原子在距离极性头 基中氧原子 $0.245 \mathrm{~nm}$ 附近出现第一个峰值, 即距离 极性头基 $0.245 \mathrm{~nm}$ 处存在第一水化层; 在 $0.472 \mathrm{~nm}$ 附近出现了相对较弱的第二峰值, 代表极性头周围 的第二水化层. 两个水化层之间的距离为 $0.227 \mathrm{~nm}$, 这一间距与分子间氢键的距离 ${ }^{31}$ 相近, 表明第二水 化层中水分子通过氢键作用与第一水化层中水分 子相连. 从表面活性剂头基和水分子的相互作用强 度可以看出, 抗衡离子为 $\mathrm{Li}^{+} 、 \mathrm{Na}^{+} 、 \mathrm{~K}^{+} 、 \mathrm{Rb}^{+}$和 $\mathrm{Cs}^{+}$时, 表面活性剂头基与第一水化层内水分子相互作用 强度变化规律为: $\mathrm{LDS}<\mathrm{SDS}<\mathrm{PDS}<\mathrm{RDS}<\mathrm{CDS}$, 为了 深入研究抗衡离子对头基与水分子相互作用的影

表3 不同体系的抗衡离子缔合度

Table 3 Counterion association degree in different systems

\begin{tabular}{cccc}
\hline System & $N_{\mathrm{f}}$ & $K_{0}$ & $N_{0}$ \\
\hline LDS & 16 & 32 & 0.50 \\
SDS & 15 & 32 & 0.47 \\
PDS & 11 & 32 & 0.34 \\
RDS & 9 & 32 & 0.28 \\
CDS & 6 & 32 & 0.19 \\
\hline
\end{tabular}

$N_{\mathrm{f}}$ : number of bridged counterions; $K_{0}$ : number of surfactants;

$N_{0}$ : counterion association degree of surfactant adsorption layer

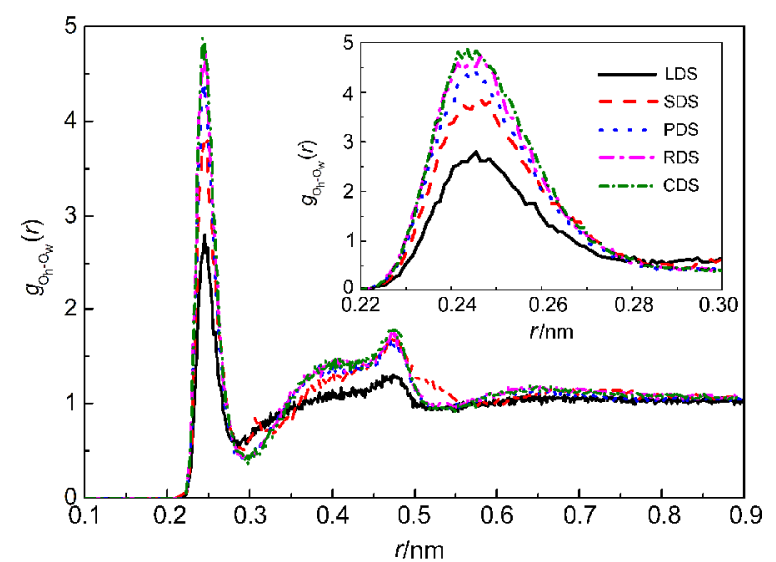

图 5 头基中氧原子 $\left(\mathrm{O}_{\mathrm{h}}\right)$ 与水中氧原子 $\left(\mathrm{O}_{w}\right)$ 的径向分布函数

Fig.5 RDF between the oxygen atom $\left(\mathrm{O}_{\mathrm{h}}\right)$ of head group and oxygen atom $\left(\mathrm{O}_{w}\right)$ of water molecules
响, 利用头基与水分子的径向分布函数计算头基周 围配位水分子数目(见图6).

从图 6 中可以看出, 5 种体系中极性头基周围水 分子的配位数由小到大的顺序为: $\mathrm{LDS}<\mathrm{SDS}<\mathrm{PDS}<$ $\mathrm{RDS}<\mathrm{CDS}$. 即随着抗衡离子半径增加, 头基与水分 子的相互作用增强, 进而导致头基周围水分子配位 数的增加. 这主要是因为随着抗衡离子半径增加, 抗衡离子进入头基周围水化层的能力减弱, 对头基 与水分子间相互作用的屏蔽能力降低. CDS 表面活 性剂头基周围水分子配位数最大, 表明 CDS 极性头 基周围水分子数目最多,头基与水分子的作用最 强. 此时, 表面活性剂单分子层稳定性最好, 体系降 低表面张力的能力最强.

\section{4 表面张力}

表面张力 ${ }^{32}$ 是描述表面结构和界面活性的基本 参量. 在分子动力学模拟中, 气/液体系的表面张力 可通过计算体系压力张量的分量获得, 具体参数可 由Kirkwood-Buff公式计算得到:

$$
\gamma_{p}=\frac{L_{z}}{2}\left[\left\langle P_{z}\right\rangle-\frac{\left\langle P_{x}\right\rangle+\left\langle P_{y}\right\rangle}{2}\right]
$$

其中, $P_{x} 、 P_{y} 、 P_{z}$ 分别是压力张量在 $x 、 y$ 和 $z$ 方向的分 量, $L_{z}$ 表示模拟体系中与气/液界面垂直的 $z$ 轴方向的 长度. 根据公式(2)所计算的不同十二烷基硫酸盐/抗 衡离子体系的表面张力如图 7 所示. 5 种体系的表面 张力大小顺序为: $\mathrm{LDS}>\mathrm{SDS}>\mathrm{PDS}>\mathrm{RDS}>\mathrm{CDS}$, 即随 着抗衡离子半径的增大, 表面活性剂降低表面张力 的能力增加. 根据软硬酸碱理论, ${ }^{33}$ 性质相似的粒子 结合能力更强. 其中, 第一主族碱金属离子柔软度 ${ }^{34}$ 的变化规律为: $\mathrm{Li}^{+}<\mathrm{Na}^{+}<\mathrm{K}^{+}<\mathrm{Rb}^{+}<\mathrm{Cs}^{+}$, 而表面活性剂

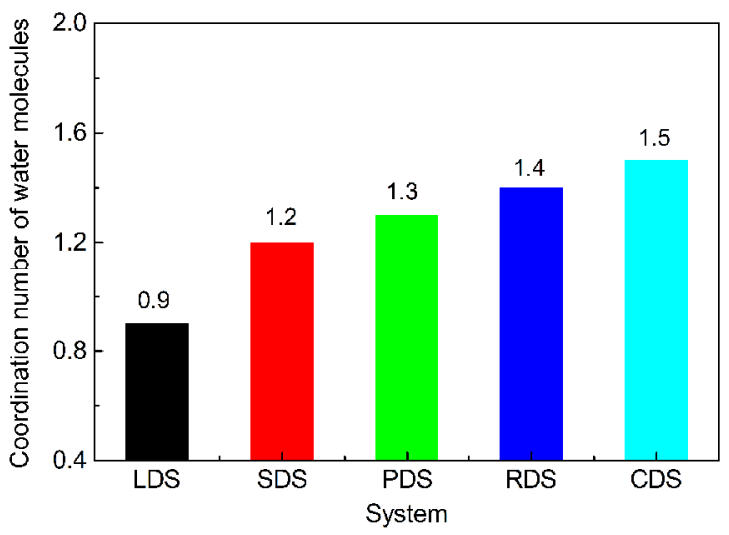

图6 表面活性剂头基周围水分子配位数

Fig.6 Coordination number of water molecules around surfactant head group 


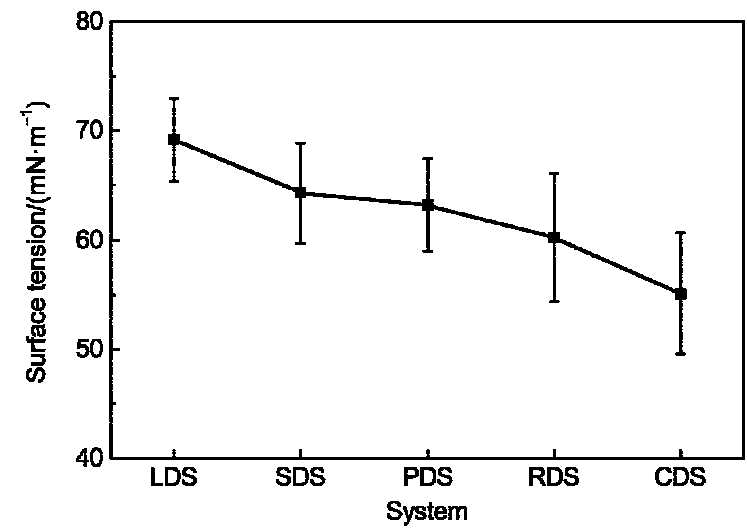

图 7 不同体系的表面张力

Fig.7 Surface tensions of different systems

极性头基硫酸根 ${ }^{35}$ 属于 “软” 离子. 因此, 抗衡离子从 $\mathrm{Li}^{+}$到 $\mathrm{Cs}^{+}$与十二烷基硫酸盐头基之间的结合能力依 次增强, 形成的分子结构稳定性依次增加, 体系的 表面张力依次降低. 该模拟结果与实验中对应的十 二烷基硫酸盐表面活性剂的表面张力 ${ }^{14}$ 的变化规律 相吻合.

\section{4 结 论}

采用分子动力学方法研究了十二烷基硫酸盐 表面活性剂气/液界面吸附行为, 并详细考察了抗衡 离子不同时, 体系在界面处的聚集状态和吸附行为 的差异.

(1) 密度曲线结果表明, 十二烷基硫酸盐表面活 性剂在气/液界面处形成单层膜, 且界面处存在明显 的界面水层结构. 抗衡离子为第一主族离子时, 随 着抗衡离子半径增加, 不同体系的界面水层厚度变 化规律为: $\mathrm{LDS}<\mathrm{SDS}<\mathrm{PDS}<\mathrm{RDS}<\mathrm{CDS}$.

(2) 径向分布函数表明, 抗衡离子为第一主族离 子时, 随着抗衡离子半径的增加不同体系 Stern 层和 扩散层厚度均依次增加, 极性头基与水分子的相互 作用强度依次增加, 且头基周围水分子配位数也随 之增大. 但吸附层的抗衡离子缔合度却随着离子半 径的增加而降低.

(3) 表面张力的结果表明, 抗衡离子为第一主族 离子时, 随着抗衡离子半径的增加, 体系表面张力 变化规律为: $\mathrm{LDS}>\mathrm{SDS}>\mathrm{PDS}>\mathrm{RDS}>\mathrm{CDS}$, 即抗衡离 子半径增加, 十二烷基硫酸盐表面活性剂表面张力 依次降低.

\section{References}

(1) Xiao, H. Y.; Zhen, Z.; Sun, H. Q.; Cao, X. L.; Li, Z. Q.; Song,
X. W.; Cui, X. H.; Liu, X. H. Acta Phys. -Chim. Sin. 2010, 26 (2), 422. [肖红艳, 甄 珍, 孙焕泉, 曹旭龙, 李振泉, 宋新旺, 崔晓红, 刘新厚. 物理化学学报, 2010, $26(2), 422$.] doi: 10.3866/PKU.WHXB20100216

(2) Che, S.; Garcia-Bennett, A. E.; Yokoi, T.; Sakamoto, K.; Kunieda, H.; Terasaki, O.; Tatsumi, T. Nat. Mater. 2003, 2 (12), 801. doi: $10.1038 /$ nmat 1022

(3) Paria, S.; Khilar, K. C. Adv. Colloid Interface Sci. 2004, 110, 75. doi: 10.1016/j.cis.2004.03.001

(4) Jang, S. S.; Lin, S. T.; Maiti, P. K.; Blanco, M.; Goddard, W. J. Phys. Chem. B 2004, 108 (32), 12130. doi: 10.1021/jp048773n

(5) Petkov, J. T.; Tucker, I. M.; Penfold, J.; Thomas, R. K.; Petsev, D. N.; Dong, C. C.; Golding, S.; Grillo, I. Langmuir 2010, 26 (22), 16699. doi: 10.1021/la1021356

(6) Manet, S.; Karpichev, Y.; Dedovets, D.; Oda, R. Langmuir 2013, 29 (11), 3518. doi: 10.1021/1a304341x

(7) Li, H. H.; Imai, Y.; Yamanaka, M.; Hayami, Y.; Takiue, T.; Matsubara, H.; Aratono, M. J. Colloid Interface Sci. 2011, 359 (1), 189. doi: 10.1016/j.jcis.2011.03.082

(8) Wang, C. Y.; Morgner, H. Phys. Chem. Chem. Phys. 2011, 13 (9), 3881. doi: 10.1039/c0cp01600d

(9) Harpham, M. R.; Ladanyi, B. M.; Levinger, N. E. J. Phys. Chem. B 2005, 109 (35), 16891. doi: 10.1021/jp0527731

(10) Schelero, N.; Hedicke, G.; Linse, P.; Klitzing, R. V. J. Phys. Chem. B 2010, 114, 15523. doi: 10.1021/jp1070488

(11) Faeder, J.; Albert, M. V.; Ladanyi, B. M. Langmuir 2003, 19 (6), 2514. doi: $10.1021 / \mathrm{la} 026755 \mathrm{w}$

(12) Wang, C. Y.; Morgner, H. Langmuir 2009, 26 (5), 3121.

(13) Bordes, R.; Tropsch, J.; Holmberg, K. J. Colloid Interface Sci. 2009, 338 (2), 529. doi: 10.1016/j.jcis.2009.06.032

(14) Gilányi, T.; Varga, I.; Mészáros, R. Phys. Chem. Chem. Phys. 2004, 6 (17), 4338. doi: 10.1039/b400958d

(15) Rakitin, A. R.; Pack, G. R. J. Phys. Chem. B 2004, 108 (8), 2712. doi: 10.1021/jp030914i

(16) Hantal, G.; Pártay, L. B.; Varga, I.; Jedlovszky, P.; Gilanyi, T. J. Phys. Chem. B 2007, 111 (7), 1769. doi: 10.1021/jp066969c

(17) Sun, H.; Ren, P.; Fried, J. R. Comput. Theor. Polym. Sci. 1998, 8 (1), 229.

(18) Li, C. X.; Li, Y.; Yuan, R.; Lv, W. Langmuir 2013, 29 (18), 5418. doi: 10.1021/la4011373

(19) Hu, X. Y.; Li, Y.; He, X. J.; Li, C. X.; Li, Z. Q.; Cao, X. L.; Xin, X.; Somasundaran, P. J. Phys. Chem. B 2011, 116 (1), 160.

(20) Tajima, K.; Muramatsu, M.; Sasaki, T. Bull. Chem. Soc. Jpn. 1970, 43, 1991. doi: 10.1246/bcsj.43.1991

(21) Yuan, S. L.; Cui, P.; Xu, G. Y.; Liu, C. B. Acta Chim. Sin. 2006, 64 (16), 1659. [苑世领, 崔 鹏, 徐桂英, 刘成卜. 化学学报, 2006, 64 (16), 1659.]

(22) Andrea, T. A.; Swope, W. C.; Andersen, H. C. J. Chem. Phys. 1983, 79, 4576. doi: 10.1063/1.446373

(23) Paterlini, M. G.; Ferguson, D. M. Chemical Physics 1998, 236 (1), 243. 
(24) Darden, T.; York, D.; Pedersen, L. J. Chem. Phys. 1993, 98 , 10089. doi: 10.1063/1.464397

(25) Yuan, S. L.; Ma, L. X.; Zhang, X. Q.; Zhang, L. Q. Colloids and Surfaces A: Physicochemical and Engineering Aspects 2006, $289(1 / 3), 2$.

(26) Yang, W. H.; Yang, X. Z. J. Phys. Chem. B 2010, 114 (31), 10069.

(27) Rivera, J. L.; McCabe, C.; Cummings, P. T. Phys. Rev. E 2003, $67(1), 011603$.

(28) Zhao, T. T.; Xu, G. Y.; Yuan, S. L.; Chen, Y. J.; Yan, H. J. Phys. Chem. B 2010, 114 (15), 5025. doi: 10.1021/jp907438x

(29) Ding, H. J.; Dai, Q. H.; Zhang, L. H.; Zhao, G. X. Acta Phys. -Chim. Sin 1994, 10, 54. [丁慧君, 戴庆红, 张君兰, 赵国秃.物理化学学报, 1994, 10, 54.] doi: 10.3866/PKU.
WHXB19940113

(30) Zhao, G. X. Physical Chemistry of Surfactants; Peking University Press: Beijing, 1984; pp 263-270. [赵国赤.表面 活性剂物理化学. 北京: 北京大学出版社, 1984: 263-270.]

(31) Yan, H.; Yuan, S. L.; Xu, G. Y.; Liu, C. B. Langmuir 2010, 26 (13), 10448. doi: 10.1021/1a100310w

(32) Cheng, T.; Chen, Q.; Li, F.; Sun, H. J. Phys. Chem. B 2010, 114 (43), 13736. doi: 10.1021/jp107002x

(33) Collins, K. D. Methods 2004, 34 (3), 309.

(34) Collins, K. D.; Neilson, G. W.; Enderby, J. E. Biophysical Chemistry 2007, 128 (2), 95.

(35) Vlachy, N.; Jagoda-Cwiklik, B.; Vácha, R.; Touraud, D.; Jungwirth, P.; Kunz, W. Adv. Colloid Interface Sci. 2009, 146 (1), 42 .

\section{5 年东方胶化杯全国胶体化学研究生优秀成果奖评选通知}

为促进中国的胶体化学发展, 鼓励这一领域青年学生的进取精神, 2002 年起特设立全国胶体化学研究 生奖学金, 以表彰优秀的胶体化学专业研究生。

\section{申请资格与范围}

全国范围内各大学、科研机构在读的胶体化学专业研究生(含博士生)均有资格申请。申请人应在胶体 与界面化学领域取得较为突出的创新性结果。发表一定数量的优秀科研论文(含已接受)。申请人的所有成 果必须是在研究生阶段进行或完成的。在职研究生不参与本奖学金评选。对于积极参加全国胶体与界面 专业委员会学术活动的科研小组,将采取适度倾斜政策给予支持。

\section{评选程序}

(1) 本人申请: 申请人需提交个人简历(含联系方式)、学术论文发表及已接受目录(含全部作者的正确顺 序、期刊、年、卷(期)、页), 发表论文的 PDF 文件, 科研工作自评, 论文工作概况。已接受文章须具备期刊的接 收函(含Email)。并说明参加胶体与界面化学专业委员会的会议情况。

(2) 单位推荐: 申请人须有本单位两名副教授以上职称的老师(含导师)推荐。各单位原则上最多推举两 名。

(3) 专家评审: 由胶体与界面化学专业委员会组织专家进行评审, 确定获奖名单。

\section{评选进程}

2015 年 3 月 1 日前递交个人申请与推荐材料(含电子版、纸版), 经专家评审后于 2015 年 4 月正式公布并 举行颁奖仪式。

联系人: 黄建滨 Tel: 010-62753557 Email: JBHuang@pku.edu.cn

邮寄地址: 北京大学化学与分子工程学院 邮政编码: 100871

本奖学金的设立获得了北京东方德菲胶体化学仪器有限公司大力支持与独家赞助。

本奖项评选工作的最终解释权在中国化学会胶体与界面化学专业委员会。 